\title{
Investigations on convective boundary layer turbulence using SODAR data
}

\author{
Francesco Tampieri and Alberto Maurizi \\ Istituto di Scienze dell'Atmosfera e del Clima (ISAC), CNR, Bologna, Italy
}

\begin{abstract}
Acoustic sounding (SODAR) data collected in convective conditions were analysed to estimate high order statistics of the vertical velocity in the lower half of the Convective Boundary Layer (CBL). Limitations of the instrumentation system were assessed and it turned out that spatial and temporal filtering have little effect on skewness and kurtosis, and do not prevent a reliable evaluation of these parameters, provided that a sufficiently long time period is analysed. Vertical profiles of skewness are grouped into two broadly defined classes, one which increases almost linearly with height and the other which shows a constant-with-height behaviour. Both behaviours are shown to be consistent with different parameterisations used in literature. Kurtosis profiles are found to be fairly well described adopting a quadratic relationship between skewness and kurtosis, provided that the correct parameterisation of skewness is used.
\end{abstract}

Key words convective boundary layer - acoustic sounding - turbulence

\section{Introduction and review}

Research on turbulence has traditionally been developed in connection with geophysics, as is shown, for instance, in the comprehensive book by Monin and Yaglom (1971, 1975), which, along with a theoretical presentation, supplies many atmospheric data. Studies on boundary layer turbulence structure made important progress from the comparison between atmospheric measurements (very high Reynolds number, fullscale size, but coarse resolution) and laboratory experiments (good control, detailed measurements, but low Reynolds number, unwanted boundary effects, reduced scale, etc.).

Mailing address: Dr. Francesco Tampieri, Istituto di Scienze dell'Atmosfera e del Clima (ISAC), CNR, Via Gobetti 101, 40129 Bologna, Italy; e-mail: f.tampieri@isac.cnr.it
Two basic shortcomings arise in the analysis and interpretation of atmospheric boundary layer turbulence data. The first refers to the intrinsic non-stationarity and inhomogeneous character of the atmosphere, leading to the impossibility of a full control of the experimental field, which contrasts with the relatively high controllability of the laboratory experiment. This corresponds to difficulties in interpreting data, but also to new stimula towards understanding the atmosphere. Nevertheless, it is possible in general to identify and single out specific aspects and study them in spite of the complexities of the atmosphere. A second difficulty, partly related to the previous one, is more practical but perhaps even more limiting. It arises from the problems in monitoring and measuring the boundary layer as completely as in the laboratory. In reality, data refer often to point measurements, made in few locations. For many years, meteorological towers constituted probably the best approach for investigating the boundary layer (for instance, the Boulder Atmospheric Observatory tower has levels of measurement up to $300 \mathrm{~m}$ above the ground). More usually, data refer to the first few meters, 
or tens of meters, above the ground. Moreover towers and masts are always intrusive, and their effects should be accounted for. Remote sensing plays an extremely useful role in overcoming part of these difficulties. In this paper, we address turbulence features in the Convective Boundary Layer (CBL) using acoustic remote soundings techniques (SODAR), examining the possibility of measuring high order statistics of vertical velocity.

Turbulence in the atmospheric CBL has specific signatures, which are important for both meteorological modelling and dispersion studies. Although most of the available experimental information and frequently used models deal with the first two moments of the velocity probability density functions $(p d f)$, the importance of skewness $S$ of the vertical component of the velocity in the CBL has long been recognised in relation to dispersion modelling (at first by Baerentsen and Berkowicz, 1984). Starting from the work of Thomson (1987), a generation of models has been designed and used, based on an appropriate estimate of the shape of the Eulerian probability density function $p d f$.

If non-Gaussianity is important, as occurs in the CBL, a knowledge of high order moments is necessary. Reliable determinations of the high order moments in atmospheric flows are difficult to achieve (Lenschow et al., 1994), and suitable parameterisations are often appropriate.

Parameterisations in the atmospheric surface layer, where both shear and buoyancy operate, are based on the similarity theory (Monin and Yaglom, 1971). Vertical profiles of the second and third moments of vertical velocity in the entire CBL have been presented by Stull (1988), Hunt et al. (1988), Weil (1990), Sawford and Guest (1987) among others. As regard fourth moments, because only very few measurements are available (Tampieri et al., 2000), a common choice is to adopt a constant profile of kurtosis $K$ (see, e.g., Du et al., 1994).

An empirical relation between skewness and kurtosis emerges quite clearly from sheardominated boundary layer data: Shaw and Seginer (1987) and Tampieri et al. (2000) have proposed a quadratic formula. A similar formula for CBL vertical velocity has been proposed by Alberghi et al. (2000).
In this paper, we discuss some results concerning skewness and kurtosis of the vertical velocity in the CBL, as derived from investigations carried out using a SODAR operated in the Rome metropolitan area, and suggest parameterisations useful for applications. Advantages and shortcomings connected to the measuring system are briefly discussed, the main objective being to explore the potential of such instruments in geophysical turbulence research, with a view to stimulate discussion on future work.

\section{Data description and treatment}

\subsection{Measurement site description}

The data used for this investigation were obtained at two sites near Rome, Italy (Pratica di Mare, hereinafter PDM, and Agenzia Municipale per l'Ambiente, hereinafter AMA) during summer 1995, 1996 and 1997, by researchers of the «Istituto di Fisica dell'Atmosfera» (IFA-CNR, Rome).

Instantaneous vertical velocity $\omega$ was measured in sampling volumes, referred to as «range gates», equally spaced with height with a $6 \mathrm{~s}$ sampling period. The radius $R$ of the horizontal section of the sampling volume varies with height $z$ as $R=z \tan \alpha$, with $\alpha$ about $9^{\circ}$. The maximum height reached is typically $700-800 \mathrm{~m}$, depending on ambient noise. The signal-to-noise ratio allows quality control on data according to Mastrantonio and Fiocco (1982) and Greenhut and Mastrantonio (1989).

\subsection{Stationarity analysis}

To obtain reliable statistics, steady time series are necessary. We selected the central part of the day, when the breeze cell due to the neighbouring coast side is well developed. Periods with clearly observable (from fac-simile) variations in the CBL thickness, in horizontal wind intensity $U$ and direction $\phi$ were excluded. Ten convective periods were singled out from this first screening.

The selected periods were subdivided into half-hour intervals for which mean $\langle w\rangle$ and 
variance $\left\langle w^{2}\right\rangle$ of vertical velocity were calculated at each height. Steadiness of first- and second-order statistics was assumed to be verified if the values lay within a band of variability of $20 \%$ for each height. Table I reports the selected periods after a further analysis of stationarity on the scaling parameters performed in the next section.

Centred moments are considered, assuming that the presence of vertical advection has a negligible influence on the CBL structure. Cases with vertical mean velocity significantly less than zero at all heights (in this study: AMA site, 1995 07-30, 1995-08-04, 1995-08-06) were attributed by Mastrantonio et al. (1994) to a displacement of the breeze cell centre.

\subsection{Error estimates}

An analysis of error sources was performed with the aim of assessing the influence of the measuring methodology on the spectrum and momentum estimates.
For the reduction of statistical error, the series with more than $15 \%$ of missing data were rejected (Greenhut and Mastrantonio, 1989) to obtain reliable estimates of high-order moments. When a time series relative to a given height was rejected, the time series for greater heights were also rejected.

Spatial and temporal filtering are of some relevance to SODAR measurements because of the nature of the technique itself. In order to estimate the effect of both filtering effects, power spectra were computed and compared to cutoff scales. Errors connected to spatial filtering were assessed according to Kristensen and Gaynor (1986). It turned out that, even in the worst case, i.e., the one that reaches the highest range gate, the correction would be of the order of the accepted error. Moreover, temporal filtering proves to be less significant than spatial filtering. It is worth pointing out that, both filtering scales being in the inertial subrange, it is reasonable to assume that the filtered part of the spectrum has less influence on the non-Gaussian properties, of interest here, than the resolved part.

Table I. Selected stationary periods in convective conditions with scaling parameter and error estimates. The subdivision is relative to the presentation in figs. 1a and $2 \mathrm{a}$ (upper part) and figs. $1 \mathrm{~b}$ and $2 \mathrm{~b}$ (lower part). Symbols on the last column are used accordingly. Reference wind is computed as a time average over the whole period and a vertical average over the selected heights.

\begin{tabular}{ccccccc}
\hline \hline Site & $\begin{array}{c}\text { Date } \\
\text { yyyy-mm-dd }\end{array}$ & $\begin{array}{c}\text { Local time } \\
\text { hh:mm }\end{array}$ & $\begin{array}{c}\text { Ref. wind } \\
\left(\mathrm{ms}^{-1}\right)\end{array}$ & $\begin{array}{c}z_{i} \\
(\mathrm{~m})\end{array}$ & $\begin{array}{c}w_{*} \\
\left(\mathrm{~ms}^{-1}\right)\end{array}$ & Symbols \\
\hline AMA & $1995-08-06$ & $10: 30-16: 30$ & 3.0 & $556 \pm 22$ & $1.24 \pm 0.03$ & $\boldsymbol{\Delta}$ \\
PDM & $1996-08-11$ & $13: 00-18: 30$ & 4.1 & $880 \pm 180$ & $1.61 \pm 0.12$ & $\square$ \\
PDM & $1997-07-01$ & $08: 30-18: 00$ & 1.9 & $652 \pm 56$ & $1.78 \pm 0.07$ & 匹 \\
PDM & $1997-08-03$ & $08: 30-18: 00$ & 3.8 & $474 \pm 25$ & $1.46 \pm 0.07$ & $\circ$ \\
PDM & $1997-08-13$ & $09: 45-13: 15$ & 2.9 & $1060 \pm 390$ & $1.93 \pm 0.26$ & $\bullet$ \\
PDM & $1997-08-13$ & $13: 15-16: 45$ & 2.9 & $529 \pm 64$ & $1.66 \pm 0.09$ & $\triangle$ \\
\hline AMA & $1995-07-30$ & $12: 00-14: 30$ & 4.5 & $1490 \pm 320$ & $1.57 \pm 0.13$ & $\square$ \\
AMA & $1995-08-04$ & $09: 30-16: 00$ & 4.5 & $1070 \pm 350$ & $1.56 \pm 0.18$ & $\square$ \\
AMA & $1995-08-15$ & $11: 30-15: 30$ & 4.8 & $1290 \pm 280$ & $1.93 \pm 0.15$ & $\circ$ \\
AMA & $1995-09-03$ & $13: 30-16: 00$ & 3.5 & $690 \pm 180$ & $1.33 \pm 0.13$ & $\bullet$ \\
PDM & $1996-07-21$ & $10: 00-16: 30$ & 5.0 & $402 \pm 27$ & $1.47 \pm 0.05$ & $\triangle$ \\
\hline
\end{tabular}


Moving on to the analysis of statistical significance, it should be noted that the number of statistically independent data for each time series is proportional to the ratio $T / \tau$, where $T$ is the total length of the time-series and $\tau$ is the Eulerian integral time-scale. Errors on estimations of moment of order $n$, due to the finite number of independent data, can be evaluated according to Lenschow et al. (1994) as $\sigma_{n} \simeq\left(k_{n} T^{-1}\right)^{1 / 2}$, where $k_{n}$ is an increasing function of the order $n$. With typical values of the process under study, a total length $T \geq 2.5 \mathrm{~h}$ is required in order to have $\sigma_{2} \leq 0.16, \sigma_{3} \leq 0.4, \sigma_{4} \leq 2.3, \sigma_{S} \leq 0.15$ and $\sigma_{K} \leq 0.6$. These could be taken as the maximum error estimates for the whole data set. Furthermore, it is worth noting that the number of independent data required for a good estimate increases with the order of the moment desired. As a last remark, it should be pointed out that relative errors on estimates of non-dimensionalised parameters $S$ and $K$ are smaller than those on third- and fourthorder velocity moments respectively.

\section{Some results concerning turbulence structure}

\subsection{Best fit estimates of $w_{*}$ and $z_{i}$}

Lacking direct measurements of the heat and momentum fluxes, the vertical profile of $\left\langle w^{2}\right\rangle$ has been used to estimate $w_{*}$ and $z_{i}$, leaving indeterminate any estimate of shear (namely, of $u_{*}$ ). Different formulations are presented in literature, which are essentially composed of two parts: one relies on the similarity in the surface layer, while the other describes the profiles for large $z / z_{i}$. Most of them can be represented by the general expression

$$
\frac{\left\langle w^{2}\right\rangle}{w_{*}^{2}}=A_{2}\left(\frac{z}{z_{i}}\right)^{2 / 3}\left(1-B_{2} \frac{z}{z_{i}}\right)^{C_{2}}
$$

where the parameters $A_{2}, B_{2}$ and $C_{2}$ assume different values according to the various authors.

The Lenschow et al. (1980) profile $\left(A_{2}=1.8\right.$, $B_{2}=0.8$ and $\left.C_{2}=2\right)$ was used to compute scaling parameters $w_{*}$ and $z_{i}$ by fitting eq. (3.1) on data.

The same profile has been used by Seibert and Langer (1996) and Melas et al. (2000) among other authors. This fitting procedure is commonly adopted (see, e.g., Weill et al., 1980; Greenhut and Mastrantonio, 1989; Seibert and Langer, 1996): since local equilibrium is better reached in the lower part of the CBL, the estimate of $w_{*}$ is expected to be more robust than that of $z_{i}$. Results of the fitting procedure on data are reported in table I.

Stationarity of $w_{*}$ and $z_{i}$ in the selected periods was further investigated by applying the fitting procedure on $\left\langle w^{2}\right\rangle$ data in half-hour sub-periods. In most cases, the scaling parameters were found to be sufficiently steady (within the error). In one case (1997-08-13), the time interval selected in Section 2 was further subdivided into two parts as reported in table I.

\subsection{Interpretation of the results}

Vertical profiles of $S$, with height non-dimensionalised by $z_{i}$, are reported in fig. $1 \mathrm{a}, \mathrm{b}$ together with some theoretical profiles from literature (Lenschow et al., 1980; Sawford and Guest, 1987; Hunt et al., 1988; Weil, 1990; Franzese et al., 1999). At first glance, it is clear that skewness data do not collapse into a unique curve, even considering the inaccuracy of the estimation of $z_{i}$ and the errors in the estimation of $S$.

It is interesting to note that $S$ profiles (both theoretical and experimental) can be grouped into two qualitatively different sets: one which shows an increase with height, at least for $z / z_{i}<0.5$, and the other which is nearly constant. This suggests that probably two quite distinct mechanisms act in shaping the vertical velocity $p d f$.

The first case corresponds to a picture of convection which organises as the height increases, giving rise to coherent vortices with maximum development near the CBL centre, where the skewness of the vertical velocity is maximum. In this case, a linear fit $S=1.2\left(z / z_{i}\right)+$ +0.1 reasonably describes the present data. On the basis of the relationship

$$
K=2.4\left(S^{2}+1\right)
$$

(Alberghi et al., 2000, 2002) a quadratic de- 

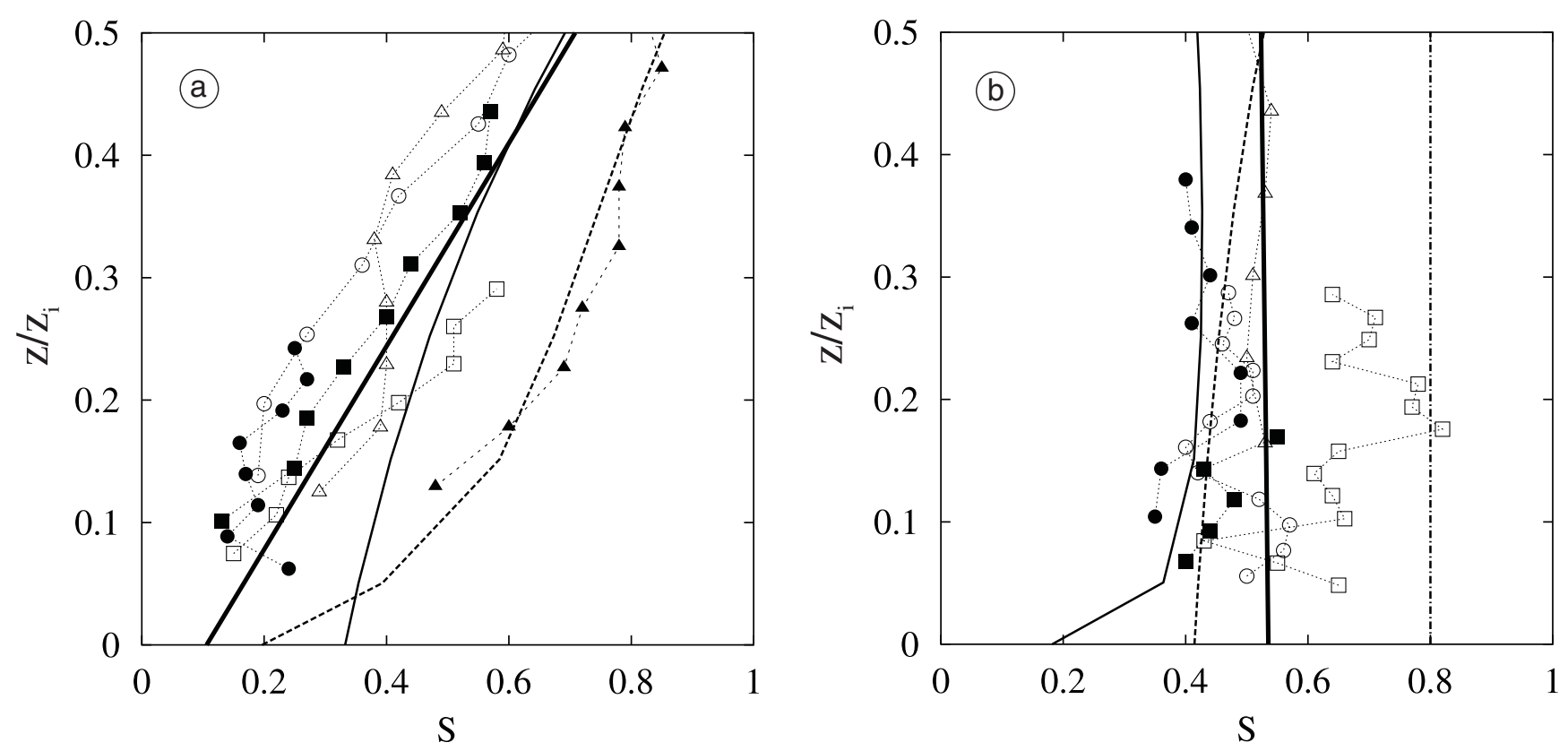

Fig. 1a,b. Vertical profiles of $S$ with height normalised with $z_{i}$, subdivided into two classes (see text). Symbols are as reported in table I. Lines are: a) thick (linear fit), solid (Lenschow et al., 1980), dashed (Weil, 1990); b) thick (linear fit), solid (Franzese et al., 1999), dashed (Hunt et al., 1988), dot-dashed (Sawford and Guest, 1987).
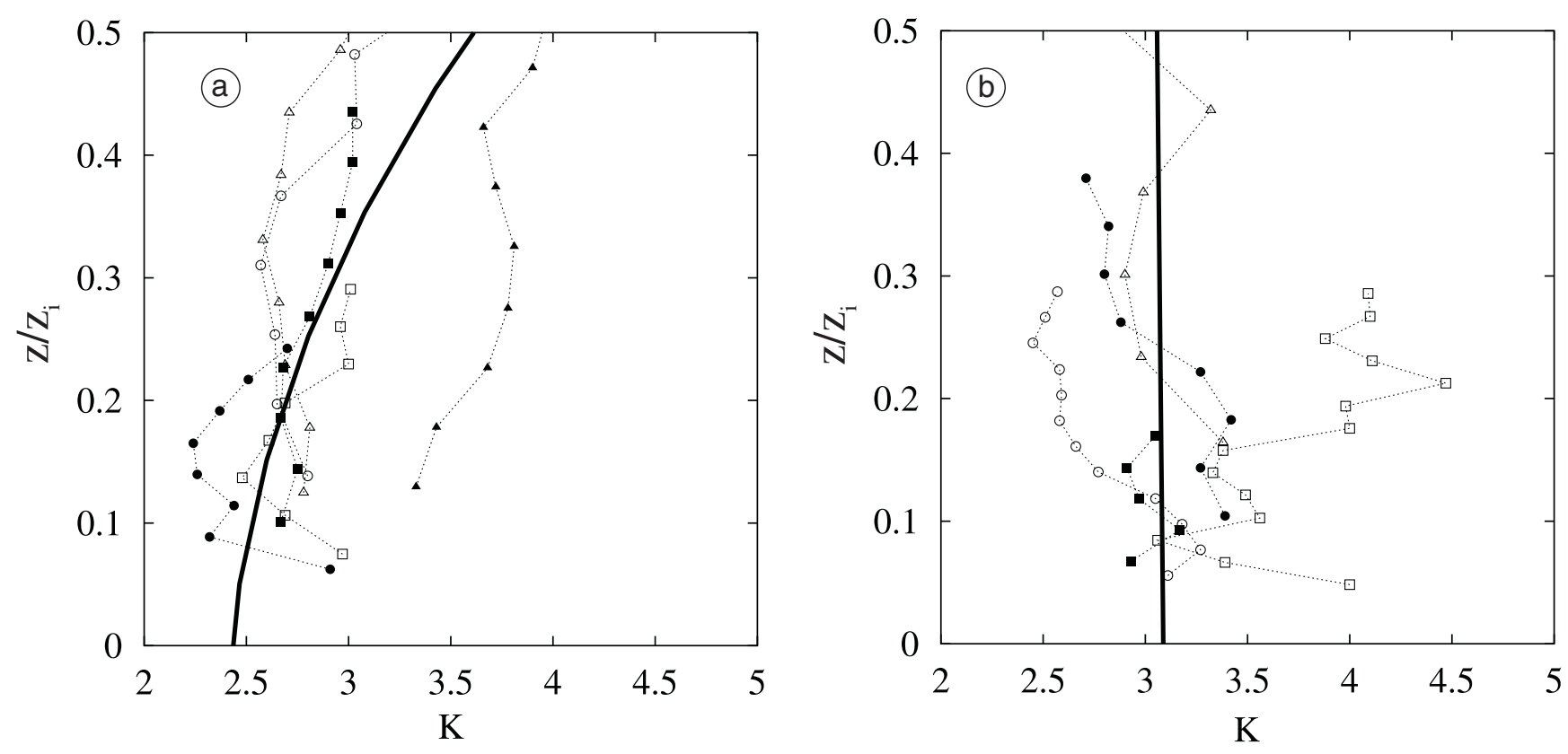

Fig. 2a,b. Vertical profiles of kurtosis. As in fig. 1a,b, symbols are as reported in table I. Lines represent eq. (3.2) with $S$ estimated by linear fit (see text and fig. 1a,b).

pendence of kurtosis from height can be established. In fig. $2 \mathrm{a}$, this relationship is plotted along with the experimental data for the same cases reported in fig. 1a.
The second case corresponds to the idea of strong mixing, leading to a homogeneous structure on the vertical. A value $S=0.5$ fits the observations. Note that in this case kurtosis is 
estimated from eq. (3.2) to be around 3, but in fact presents a large variability (see fig. 2b).

We note in passing that the grouping of profiles evidences that they mostly behave differently in the two sites. The AMA site shows a large scatter and a nearly constant with height behaviour, while PDM displays a more coherent increase with height.

\section{Conclusions}

In the present paper SODAR data were analysed to give a picture of the convective boundary layer turbulence on almost flat terrain, in synoptic calm conditions. The main objective of the work was to investigate the possibility of obtaining reliable evaluations of some turbulence parameters (moments of 3rd and 4th order, spectra) through remote sensing techniques. In summary, it turns out that the limitations due to the finite volume of sampling, and the relatively low time resolution, do not strongly affect the capability of producing robust and consistent statistics.

In particular, two different situations were disclosed, reflecting conditions of vertical homogeneity or not in the CBL. Moreover, the existence of a quite universal relationship between skewness and kurtosis (well established in shear dominated boundary layers and poorly documented in the CBL) can account for vertical profiles of kurtosis if a reasonable parameterisation for $S$ is given.

The authors would like to close by recalling the pioneering work conducted by Giorgio Fiocco in the development of remote sensors like SODAR, which in fact allowed the present work to be carried out. The senior author was impressed by a paper by Fiocco and Mastrantonio (1983) where an investigation on the structure of the flow around the top of Monte Rosa was reported, using a SODAR located near the mountain top. The paper suggested the possibility of performing studies of atmospheric turbulence statistics in a much easier and more efficient way with respect to punctual measuring devices (i.e. sonic anemometers). The present work shows an application of such ideas in a relatively simple case; we close with the hope of a detailed docu- mentation of turbulent flow in complex terrain, using remote sensors, as an important step along the path initiated at Monte Rosa some 20 years ago.

\section{REFERENCES}

ALBERGHI, S., F. TAMPIERI, S. ARGENTINI, G. MASTRANTONIO and A. VIOLA (2000): Analysis of the $p d f$ of the vertical velocity in the buoyancy-driven atmospheric boundary layer, Advances in Turbulence VIII, Proceedings of the Eighth European Turbulence Conference, edited by C. DOPAZO (CIMNE, Barcelona), 315-318.

Alberghi, S., A. MAurizi and F. TAMPIERI (2002): Observations on the skewness-kurtosis relationship of vertical velocity in the convective boundary layer, $J$. Appl. Meteorol., 41, 885-889.

BAERENTSEN, J.H. and R. BERKOWICZ (1984): Monte-Carlo simulation of plume diffusion in the convective boundary layer, Atmos. Environ., 18, 701-712.

DU, S., J.D. WILSON and E. YeE (1994): Probability density functions for velocity in the convective boundary layer, and implied trajectory models, Atmos. Environ., 28, 1211-1217.

FIOCCO, G. and G. MASTRANTONIO (1983): Characters of the air flow inferred from detailed spectral analysis of acoustic sounder echoe, J. Acoust. Soc. Am., 74, 1861-1865.

Franzese, P., A.K. LuHAR and M.S. Borgas (1999): An efficient lagrangian stochastic model of vertical dispersion in the convective boundary layer, Atmos. Environ., 33, 2337-2345.

GREENHUT, G.K. and G. MASTRANTONIO (1989): Turbulence kinetic energy budget profiles derived from Doppler sodar measurements, J. Appl. Meteorol., 28, 99-106.

HunT, J.C.R., J.C. KAIMAL and J.E. GAYNOR (1988): Eddy structure in the convective boundary layer-new measurements and new concepts, Quart. J. R. Meteorol. Soc., 114, 827-858.

KRISTENSEN, L. and J.E. GAYNOR (1986): Errors in second moments estimated from mopnostatic Doppler sodar winds. Part I: theoretical description, J. Atmos. Ocean. Tecnol., 3, 523-528.

LENSCHOW, D.H., J.C. WyngaARD and W.T. PENNELL (1980): Mean-field and second-moment budgets in a baroclinic, convective boundary layer, J. Atmos. Sci., 37, 1313-1326.

LENSCHOW, D.H., J. MANN and L. KRISTENSEN (1994): How long is long enough when measuring fluxes and other turbulence statistics?, J. Atmos. Oceanic Technol., 11, 661-673.

Mastrantonio, G. and G. FiocCo (1982): Accuracy of wind velocity determinations with Doppler sodar, $J$. Appl. Meteorol., 21, 820-830.

Mastrantonio, G., A. Viola, S. Argentini, G. Fiocco, L. Giannini, L. Rossini, G. AbBAte, R. OCONE and M. CASONATO (1994): Observations of sea breeze events in Rome and the surrounding area by a network of Doppler 
sodars, Boundary-Layer. Meteorol., 71(1-2), 67-80.

Melas, D., G. Abbate, D. Haralampopoulos and A. KeLESIDIS (2000): Estimation of meteorological parameters for air quality management: coupling of SODAR data with simple numerical models, J. Appl. Meteorol., 39, 509-515.

Monin, A.S. and A.M. YAGLOM (1971): Statistical Fluid Mechanics, edited by J. LuMLEY (MIT Press, Cambridge), vol. 1, pp. 769.

Monin, A.S. and A.M. YAGLOM (1975): Statistical Fluid Mechanics, edited by J. LuMLEY (MIT Press, Cambridge), vol. 2, pp. 874.

SAWFORD, B.L. and F.M. Guest (1987): Lagrangian stochastic analysis of flux gradient relationships in the convective boundary layer, J. Atmos. Sci., 44, 1152-1165.

SEIBERT, P. and M. LANGER (1996): Deriving characteristic parameters of the convective boundary layer from SODAR measurements of the vertical velocity variance, Boundary-Layer. Meteorol., 81, 11-22.

SHAW, R.H. and I. SEGINER (1987): Calculation of velocity skewness in real and artificial plant canopies, BoundaryLayer. Meteorol., 39, 315-332.

StULL, R.B. (1988): An Introduction to Boundary Layer Meteorology (Kluwer Academic Publishers, Dordrecht), pp. 666.

TAMPieri, F., A. Maurizi and S. Alberghi (2000): Lagrangian models of turbulent dispersion in the atmospheric boundary layer, Ingegneria del Vento in Italia 2000, edited by G. SOLARI, L.C. PAGNINI and G. PICCARDO (SGEditoriali, Padova), 37-50.

THOMSON, D.J. (1987): Criteria for the selection of stochastic models of particle trajectories in turbulent flows, J. Fluid Mech., 180, 529-556.

WEIL, J.C. (1990): A diagnosis of the asymmetry in top-down and bottom-up diffusion using a Lagrangian stochastic model, J. Atmos. Sci., 47 (4), 501-515.

Weill, A., C. Klapisz, B. Strauss, F. Baudin, C. JAUPART, P. VAN GRUDERBEEK and J.P. GOUTORBE (1980): Measuring heat flux and structure functions of temperature fluctuations with an acoustic Doppler sodar, J. Appl. Meteorol., 19, 199-205. 